\title{
Anastomose mecânica esofagogástrica cervical látero-lateral após esofagectomias
}

\section{Mechanical cervical esophagogastric laterolateral anastomosis after esophagectomies}

João Batista Neto, TCBC-Al'; Paula Gabriela Melo Morais²; Marcos da Costa Nepomuceno, ACbC-Al³; José Cardoso

Cavalcante JR, TCBC-AL ; Tadeu Muritiba, TCBC-Al ${ }^{3}$; Alberto Jorge Fontan, TCBC-Al ${ }^{5}$

R E S U M O

\begin{abstract}
Dentre os desafios das esofagectomias, a fístula da anastomose cervical persiste desafiadora por suas consequências, sendo a principal, a estenose da anastomose. Os autores apresentam uma técnica de anastomose esofagogástrica cervical látero-lateral mecânica à Orringer, que reduz o índice de fístulas e de estenose.
\end{abstract}

Descritores: Esôfago. Esofagectomia. Fístula anastomótica. Anastomose cirúrgica. Técnica de fechamento de ferimentos abdominais.

\section{INTRODUÇÃO}

D entre os desafios das esofagectomias, a fístula da anastomose cervical continua um dos problemas principais, por sua morbimortalidade, dentre os quais se destaca a estenose, pelas restrições ou sofrimento que impõe ao doente.

A fístula cervical incide em 10 a $50 \%$ das anastomoses e a metade resulta em estenose com consequente necessidade de sessões de dilatação'.

Em 1998, Collard et al. ${ }^{2}$, compararam uma nova técnica de anastomose esofagogástrica cervical (AEGC) látero-lateral mecânica, utilizando a parte mais superior do fundo gástrico e a parede posterior do esôfago, com a clássica esofagogastrostomia término-lateral, através de sutura manual. Seu intuito era reduzir o alto risco de fístula e estenose que acarretavam. Seus resultados com uso do grampeador na modalidade de sutura látero-lateral foram satisfatórios ao reduzir o índice de estenose com a nova técnica.

Orringer et al. ${ }^{1}$, em 2000, modificaram esta técnica e demonstraram sua eficiência, realizando uma anastomose mecânica látero-lateral, porém utilizando a parede anterior do estômago e a posterior do esôfago. Dessa forma minimizaria o refluxo gastroesofágico ao criar uma bolsa gástrica retroesofágica que se distende com ar à deglutição. Esta técnica reduziu seu índice de fístula de 10 a $15 \%$, quando utilizava sutura manual, para $2,7 \%$ de fístulas clinicamente expressivas ao realizar essa nova anastomose com grampeador.

O objetivo deste estudo é divulgar a técnica de Orringer et al. ${ }^{1}$, em pacientes submetidos à esofagectomia trans-hiatal, aplicando-a no estômago transposto ou tubulizado, com resultados satisfatórios, devido à ausência de publicações sobre a mesma entre os cirurgiões brasileiros.

\section{TÉCNICA}

A esofagectomia transhiatal ${ }^{3}$ é realizada através dos acessos abdominal e cervical esquerdo. É indicada no megaesôfago avançado, nos tumores do terço inferior do esôfago, do cárdia ou estenoses de doenças benignas. Todas as figuras foram adaptadas de Orringer et al. ${ }^{1}$.

Realizado o acesso cervical, com o esôfago dissecado e estômago (completo ou tubulizado) pronto para ser transposto, prosseguimos realizando: 1 - tração delicada da peça cirúrgica até que o estômago se exteriorize $5 \mathrm{~cm}$ acima da clavícula esquerda (Figura 1). Nesta passagem transmediastinal, o estômago, deve ser monitorado, para que não fique rodado em torno de seu eixo; 2- a secção da transição esofagogástrica com grampeador linear cortante TLC75. Realiza-se a sutura manual de re-

\footnotetext{
Trabalho realizado no Grupo de Cirurgia do Esôfago, Estômago e Cirurgia Bariátrica do Serviço de Cirurgia Geral e Especialidades do Hospital Universitário Prof. Alberto Antunes(HUPAA), da Faculdade de Medicina da Universidade Federal de Alagoas(FAMED/UFAL), Maceió.

1. Prof. Adjunto-Mestre do Módulo Digestório da Disciplina Saúde do Adulto e do Idoso da Faculdade de Medicina da Universidade Federal de Alagoas; 2. Residente de Cirurgia Geral do Serviço de Cirurgia Geral e Especialidades do Hospital Universitário Prof. Alberto Antunes; 3. Assistente do Serviço do Serviço de Cirurgia Geral e Especialidades do Hospital Universitário Prof. Alberto Antunes; 4. Diretor Técnico do Centro de Alta Complexidade em Oncologia Prof. Úlpio Miranda; 5. Chefe do Serviço de Cirurgia Geral e Especialidades do Hospital Universitário Prof. Alberto Antunes.
} 
forço, contínua, ancorada, com poliglecaprone 3-0; 3- liberando o coto esofágico remanescente, até que, deite sua face posterior sobre a parede anterior do estômago, pouco redundante, para que ao tracionar a estrutura reconstituída, a anastomose não fique tensa; 4- reparo das bordas esofágicas, das bordas do fundo gástrico, do estômago no corpo, para que fique estendido (Figura 2). Realiza-se gastrostomia na parede anterior do estômago com bisturi elétrico, em extensão de $1 \mathrm{~cm}$, o suficiente para caber a pá inferior do grampeador numa extensão de, pelo menos, $3 \mathrm{~cm}$, para ficar uma boa boca anastomótica. Aposição da pá anterior do grampeador. Ajuste das duas pás e disparo da sutura mecânica com leve inclinação do grampeador para a direita. Sutura de três pontos entre a seromuscular do estômago e muscular esofágica em ambos os lados (Figura 3). Passagem de sonda enteral ou nasogástrica 16F, para alimentar 24 horas após, deixando-a no duodeno; 5- sutura manual contínua, ancorada, da face anterior da anastomose esofagogástrica com poliglecaprone 3-0, iniciando-se com fio em cada lado e os dois se encontrando no meio da sutura. Faz-se um segundo plano de sutura com pontos separados para inversão da sutura (Figura 4); 6- uma tração suave do estômago na cavidade abdominal, para aposição adequada da anastomose na região cervical e fixam-se dois pontos na transição hiatal e na fáscia prévertebral. Drenagem da região cervical com Penrose $n^{\circ}$ 1. Fechamento por planos.

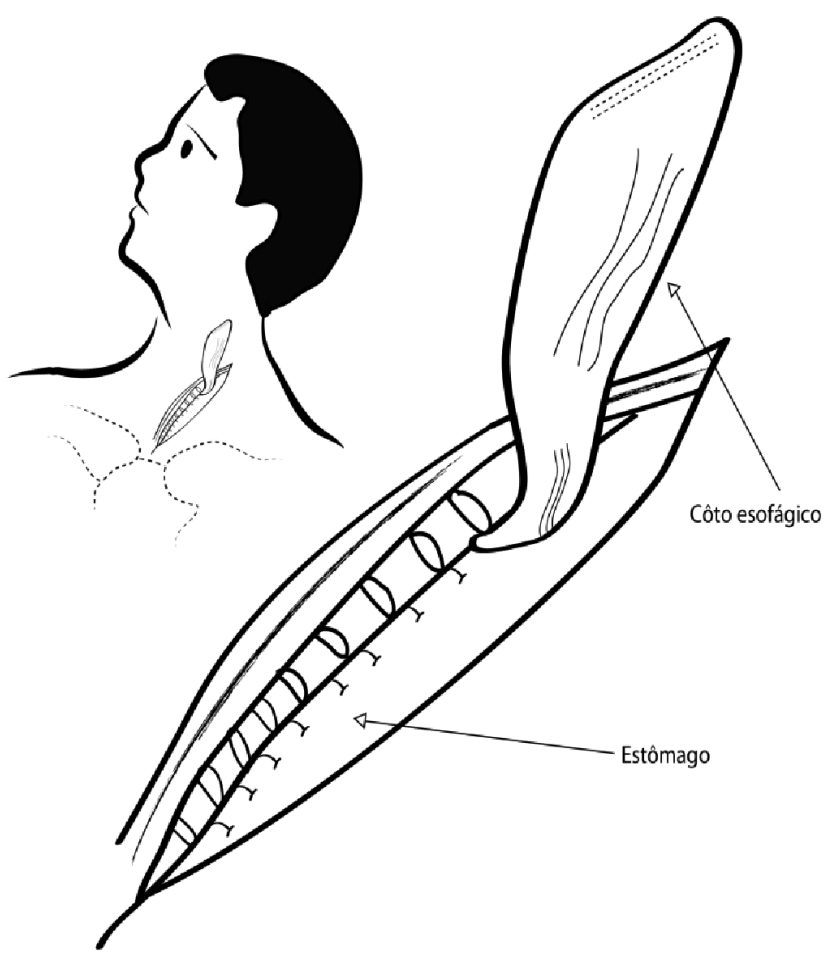

Figura 1 - Coto esofágico dissecado. Estômago completo ou tubulizado transposto.

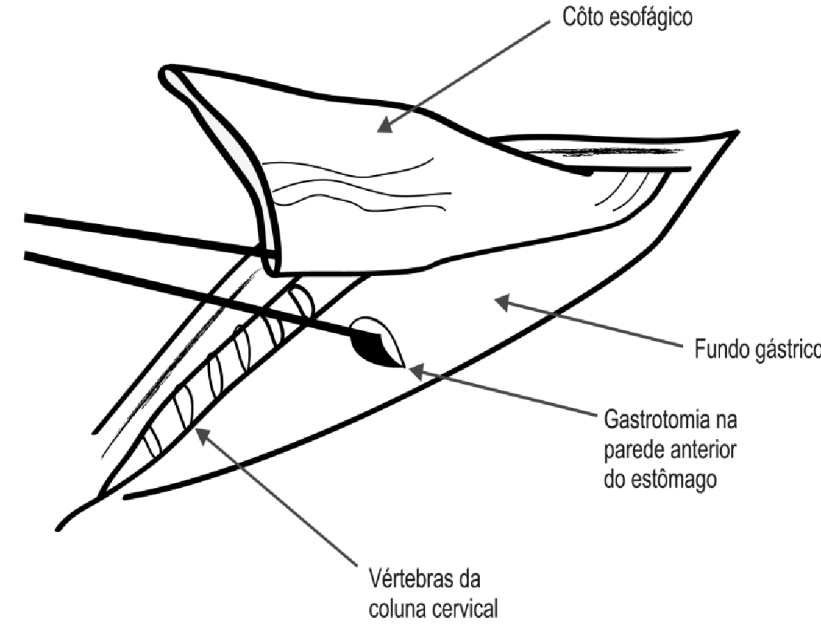

Figura 2 - Gastrotomia transversa de $1 \mathrm{~cm}$ na parede anterior do estômago.

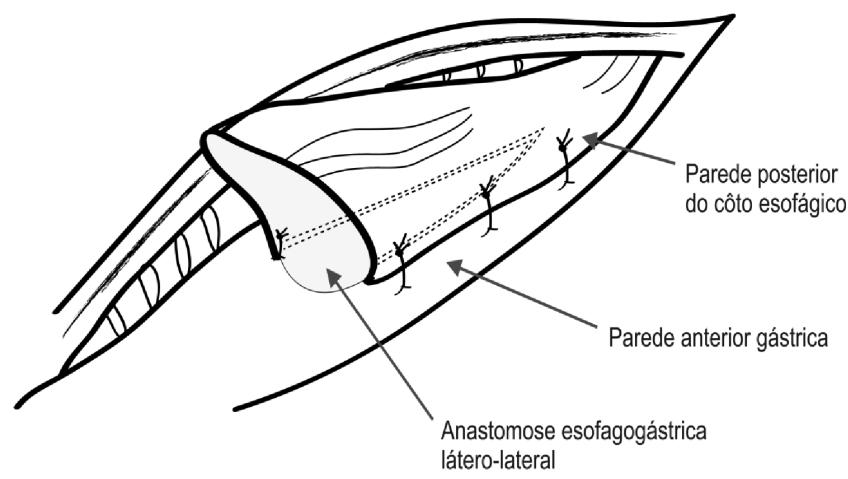

Figura 3 - Grampeamento com TLC75 entre a parede posterior do esôfago e a parede anterior do estômago.

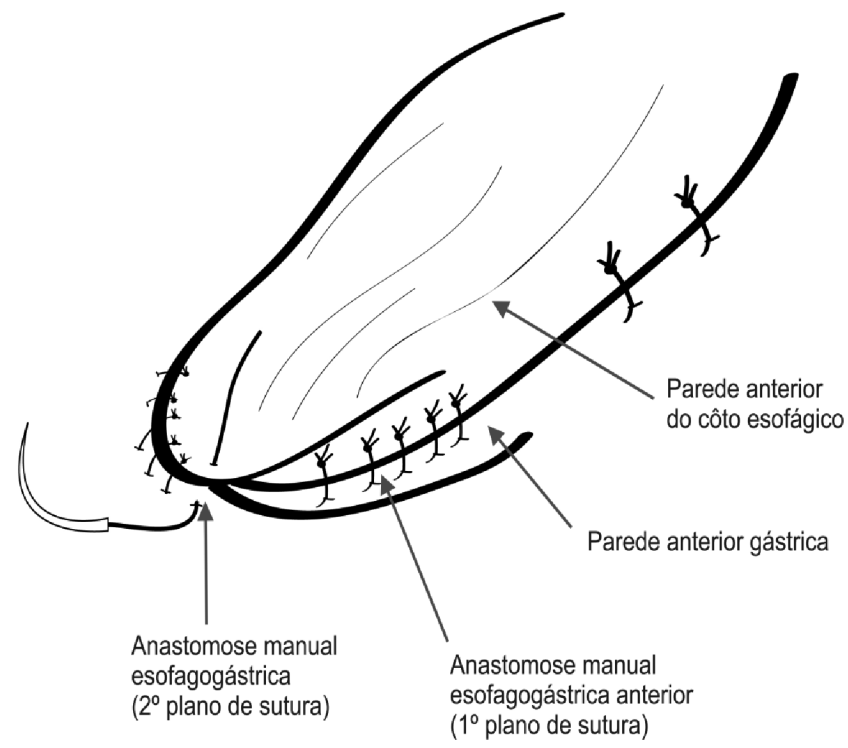

Figura 4 - Anastomose manual esofagogástrica látero-lateral, em dois planos, sendo o primeiro em sutura contínua com poliglecaprone 3-0 e a segunda invaginante em pontos separados. 


\section{DISCUSSÃO}

A experiência do Serviço com esofagectomia trans-hiatal foi iniciada em 1982 e gira em torno de uma centena, para doenças benignas e malignas através de acesso aberto ou laparoscópico. A incidência de fístulas foi $32 \%$ no início e reduziu-se para $13,3 \%$, tendo $25 \%$ dos casos de fístula necessitado de uma a três sessões de dilatação ${ }^{4}$.

Múltiplas tentativas de técnicas são relacionadas na literatura nacional e estrangeira, sem solução da questão, visto que, quando reduzem o índice de fístulas não o fazem quanto à estenose ou vice-versa ${ }^{1,2,5-8}$. O acesso laparoscópico não alterou o índice de fístulas quando comparado ao acesso aberto ${ }^{9}$.

Em 2012, Saluja et al..$^{10}$, em estudo prospectivo randomizado, encontraram índice similar de fístulas quando comparadas as suturas manual versus mecânica. No entanto, o índice de estenose se reduziu significativamente na técnica com grampeador.
Vivendo a mesma angústia dos cirurgiões que praticam a cirurgia esofágica, adotamos a inovação de Orringer et al. ${ }^{1}$, adaptando-a à realidade brasileira, utilizando o TL75, substituindo o endoGIA original e obtendo resultados satisfatórios com a anastomose mecânica sobre a manual, desde 2008. Até o momento, não houve nenhuma fístula. Apenas uma subestenose à imagem radiológica, sem expressão clínica. O acompanhamento dos doentes se fez por período que vai até 48 meses e no seguimento endoscópico o achado foi de boca ampla da anastomose e sem refluxo para o coto esofágico.

Concluímos que a técnica, adotada no nosso Serviço, é promissora, porém sua validação depende de estudos longitudinais prospectivos com casuística comparada adequada.

\section{Agradecimento}

Ao designer gráfico Jorge Luiz Ferreira Carlos, pela adaptação das ilustrações.

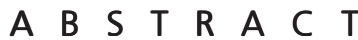

Among the challenges of esophagectomies, the cervical anastomosis remains challenging for its consequences, the main being the anastomotic stricture. The authors present a technique for an Orringer-fashion mechanical cervical laterolateral esophagogastric anastomosis, which reduces the rate of fistula and stenosis.

Key words: Esophagus. Esophagectomy. Anastomotic leak. Anastomosis, surgical. Abdominal wound closure techniques.

\section{REFERENCIAS}

1. Orringer MB, Marshall B, lannettoni MD. Eliminating the cervical esophagogastric anastomotic leak with a side-to-side stapled anastomosis. J Thorac Cardiovasc Surg. 2000;119(2):277-88.

2. Collard JM, Romagnoli R, Goncette L, Otte JB, Kestens PJ. Terminalized semimechanical side-to-side suture technique for cervical esophagogastrostomy. Ann Thorac Surg. 1998;65(3):8147.

3. Ferreira EAB. Esofagectomia subtotal e esofagogastroplastia transmediastinal posterior sem toracotomia no tratamento do megaesôfago [tese de livre-docência]. São Paulo: Faculdade de Medicina, Universidade de São Paulo; 1975.

4. Batista Neto J, Fontan AJ, Nepomuceno MC, Lourenço LG, Ribeiro LT, Ramos CP. Esofagectomia trans-hiatal no tratamento do megaesôfago chagásico avançado. Rev Col Bras Cir. 2003;30(3):230-7.

5. Kunzle JE, Ziliotto Júnior A. Estudo das anastomoses esofágicas cervicais após esofagectomia. Rev Col Bras Cir. 1985;12(2):42-6.

6. Pinotti HW, Cecconelo I, da Rocha JM, Zilberstein B. Resection for achalasia of the esophagus. Hepatogastroenterology. 1991;38(6):470-3

7. Aquino JLB, Camargo JGT, Said MM, Merhi VAL, Maclellan KCP, Palu BF. Avaliação da anastomose esofagogástrica cervical com sutura mecânica e manual em pacientes com megaesôfago avançado. Rev Col Bras Cir. 2009;36(1):19-23.
8. Henriques AC, Zanon AB, Godinho CA, Martins LC, Saad Júnior R, Speranzini $M B$, et al. Estudo comparativo entre as anastomoses cervicais esofagogástrica término-terminal com e sem invaginação após esofagectomia para câncer. Rev Col Bras Cir. 2009;36(5):398405.

9. Crema E, Ribeiro LB, Terra JA Jr, Silva AA. Laparoscopic transhiatal subtotal esophagectomy for the treatment of advanced megaesophagus. Ann Thorac Surg. 2005;80(4):1196-201.

10. Saluja SS, Ray S, Pal S, Sanyal S, Agrawal N, Dash NR, et al. Randomized trial comparing side-to-side stapled and hand-sewn esophagogastric anastomosis in neck. J Gastrointest Surg. 2012;16(7):1287-95

Recebido em 20/08/2012

Aceito para publicação em 15/10/2012

Conflito de interesse: nenhum

Fonte de financiamento: nenhuma

\section{Como citar este artigo:}

Batista Neto J, Morais PGM, Nepomuceno MC, Cavalcante Júnior JC, Muritiba T, Fontan AJ. Anastomose mecânica esofagogástrica cervical látero-lateral após esofagectomias. Rev Col Bras Cir. [periódico na Internet] 2013;40(5). Disponível em URL: http://www.scielo.br/rcbc

\section{Endereço para correspondência:}

João Batista Neto

E-mail: www.jbatista19.jb@gmail.com 